\title{
Mesial temporal lobe morphology in intractable pediatric epilepsy: so-called hippocampal malrotation, associated findings, and relevance to presurgical assessment
}

\author{
James L. Leach, MD, ${ }^{1}$ Reem Awwad, MD, ${ }^{1}$ Hansel M. Greiner, MD, ${ }^{2}$ Jennifer J. Vannest, PhD, ${ }^{2}$ \\ Lili Miles, MD, ${ }^{4}$ and Francesco T. Mangano, $\mathrm{DO}^{3}$ \\ Departments of ${ }^{1}$ Radiology, ${ }^{2}$ Neurology, ${ }^{3}$ Neurosurgery, and ${ }^{4}$ Pathology, Comprehensive Epilepsy Treatment Center, Cincinnati \\ Children's Hospital Medical Center, Cincinnati, Ohio
}

\begin{abstract}
OBJECTIVE Diagnostic criteria for hippocampal malrotation (HIMAL) on brain MRI typically include a rounded hippocampus, vertical collateral sulcus, and architectural blurring. Relationship to epileptogenesis remains speculative, and usefulness for surgical guidance is unknown. The study was performed to determine the prevalence of hippocampal rotational anomalies in a cohort of pediatric patients with intractable epilepsy undergoing evaluation for surgery and to determine the significance of this finding in the context of surgical planning.

METHODS Forty-eight surgically treated children with intractable epilepsy were compared with matched healthy subjects; reviewers were blinded to surgical side. Each temporal lobe was evaluated for rounded hippocampus, blurring, vertical collateral sulcus, wide choroidal fissure, enlarged temporal horn, low fornix, hippocampal signal, and findings of hippocampal sclerosis. A mesial temporal lobe (MTL) score was calculated by summing the number of features, and the collateral sulcus angle (CSA) was measured in each temporal lobe. Surgical side, pathological diagnosis, and imaging findings elsewhere in the brain were tabulated. Presence of HIMAL, associated imaging features, and MTL score were compared between sides, between epilepsy and control groups, in relationship to side of surgery, and in relationship to postoperative outcome.
\end{abstract}

RESULTS Only 3 epilepsy patients (6.2\%) and no controls exhibited all 3 features of HIMAL ( $p=0.12$ ). Eight of 48 (16.7\%) epilepsy versus 2 of $48(4.6 \%)$ control subjects had both a rounded hippocampus and vertical collateral sulcus (suggesting HIMAL) ( $p=0.045$ ). In control and epilepsy subjects, most findings were more prevalent on the left, and the left CSA was more vertical $(p<0.0001)$. Epilepsy subjects had higher MTL scores $(z=-2.95, p=0.002)$ and more acute CSAs $(p=0.04)$ than controls. Only lateralizing raw MTL score had a significant association with surgical side $(p=$ 0.03 , OR 7.33); however, this was not significant when hippocampal sclerosis cases were excluded. HIMAL findings were more prevalent and MTL scores were higher in patients with resections involving the temporal lobes. On group analysis, HIMAL findings did not predict eventual surgical side and did not predict outcome, although the numbers are small. In 4 patients the abnormally rotated hippocampus was resected and showed hippocampal sclerosis and/or dysplastic changes on histopathology. All of these patients had a good outcome after surgery.

CONCLUSIONS While increased in prevalence in children with intractable epilepsy, imaging findings of HIMAL did not have preoperative lateralizing utility in this group. Findings of HIMAL (including round hippocampus, architectural blurring, and vertical collateral sulcus) did not predict outcome after surgery, although the small number of patients with these findings limits evaluation. In the small number of patients in which the malrotated hippocampus was removed, outcome was good. Further research is needed to continue to define this association in children with intractable epilepsy, focusing on a temporal lobe cohort.

http://thejns.org/doi/abs/10.3171/2015.11.PEDS15485

KEY WORDS hippocampal malrotation; HIMAL; epilepsy; children; surgery

ABBREVIATIONS ATAH = anterior temporal lobectomy and amygdalohippocampectomy; $C A=$ cornu ammonis; $C D=$ cortical dysplasia; $C-M I N D=$ Cincinnati $M R$ Imaging of NeuroDevelopment; $\mathrm{CSA}=$ collateral sulcus angle (angle of the collateral sulcus, relative to the perpendicular falx); EEG $=$ electroencephalography; $E T R=$ extratemporal resection; HIMAL = hippocampal malrotation; ILAE = International League Against Epilepsy; $\mathrm{LI}$ = lateralization index; $\mathrm{MTL}=$ mesial temporal lobe; PACS = picture archiving and communication system; TLR = temporal lobe resection.

SUBMITTED August 10, 2015. ACCEPTED November 2, 2015.

INCLUDE WHEN CITING Published online February 12, 2016; DOI: 10.3171/2015.11.PEDS15485. 
$\mathrm{A}$ ROUNDED appearance of the hippocampus associated with other potentially related ipsilateral structural findings on MRI has been thought to be increased in prevalence in patients with epilepsy. ${ }^{1,12}$ Some studies have also demonstrated an increased prevalence of this morphology in children with febrile status epilepticus, suggesting that there may be an etiological link, ${ }^{31}$ although this has recently been questioned. ${ }^{9}$ This morphology has been variously described depending on the defining criteria chosen in each study (e.g., "hippocampal malrotation," or HIMAL, "incomplete hippocampal inversion," or "hippocampal morphologic modification"). ${ }^{1,2,4,5,12,20}$ Imaging criteria for HIMAL have varied but have typically included asymmetrical medialized rounded hippocampal shape, vertical collateral sulcus, internal architecture blurring, and ipsilateral low fornix position. ${ }^{1,2,12}$ Prior studies have reported a wide variation in HIMAL prevalence, from $1 \%-19 \%$ in healthy controls to $6 \%-30 \%$ in patients with epilepsy (mostly adult studies), ${ }^{1,2,5,31}$ with the highest prevalence in significant anomalies of brain development (64\%-85\%). 5,21,29 This suggests that HIMAL is a developmental rather than an acquired lesion.

The prevalence of HIMAL in the healthy pediatric population and in children with intractable epilepsy has not been well studied, and its relationship to epileptogenesis remains unknown. Given the known changes of hippocampal morphology that occur during development, ${ }^{25,33}$ assessing a pediatric cohort is important. In pediatric epilepsy patients undergoing surgical therapy, MRI findings may be normal or nonlesional in approximately $50 \%$ of cases. ${ }^{22}$ Any imaging finding that might help in lateralization would be useful in these patients. We could find no prior study assessing the lateralizing value of HIMAL in a surgical cohort.

Our main questions were 1) What is the prevalence of hippocampal rotational anomalies in a pediatric cohort with intractable epilepsy undergoing evaluation for surgery? 2) Is there any lateralizing significance of this finding that can assist in surgical planning?

\section{Methods}

This study was approved by our institutional review board. The epilepsy surgery database was searched between January 2007 and December 2012 for patients with intractable epilepsy who underwent resection. Patients with tumors, temporal lobe destructive lesions, gross gyral malformations involving the temporal lobe (polymicrogyria, lissencephaly), callosal agenesis, or inadequate imaging were excluded. After exclusion, 48 consecutive surgically treated children with intractable epilepsy formed the study group. Subjects were evaluated by a dedicated seizure protocol MRI, video-electroencephalography (EEG) monitoring, PET, SISCOM, and magnetoencephalography as part of the standard epilepsy surgery workup at our institution in concert with a multidisciplinary conference as previously described. ${ }^{30}$ All subjects underwent imaging following a dedicated seizure protocol MRI, as detailed elsewhere. ${ }^{23,30}$ Forty-four patients underwent imaging at 3 $\mathrm{T}$ and 4 patients at $1.5 \mathrm{~T}$ using $1-\mathrm{mm}(\mathrm{n}=46)$ or $1.25-\mathrm{mm}$ $(\mathrm{n}=2)$ isotropic T1-weighted sequences, and 1-mm isotro- pic T2-weighted FLAIR volumetric sequences $(n=41)$. A matched control group of 48 healthy children was obtained using Cincinnati MR Imaging of NeuroDevelopment (CMIND) study group subjects who underwent MRI at our institution. ${ }^{16,34}$ C-MIND is a publicly accessible imaging database of healthy children specifically excluding those with neurological or psychiatric illness (including seizures) in the subjects or their first-degree relatives, and mandating normal development and IQ higher than 80 (in children 5 years or older). ${ }^{34}$ The imaging protocol for the C-MIND study is described in detail elsewhere ${ }^{16}$ and included 1-mm isotropic T1-weighted and T2-weighted FLAIR volumetric sequences performed at $3 \mathrm{~T}$, identical to those performed as part of the 3-T epilepsy protocol performed on the clinical cohort. HIMAL was not used as a lateralizing lesional finding for epilepsy surgery. ${ }^{23}$ The control population was matched as closely as was feasible with the study population with regard to age, sex, and handedness. Resection and pathological analysis were performed as described elsewhere. 23 Pathological classification used the 2011 International League Against Epilepsy (ILAE) classification system for cortical dysplasia. ${ }^{6}$

To assess the various morphological features and measurements of the temporal lobe, volumetric 3D T1-weighted and T2-weighted FLAIR data sets were analyzed in a clinical picture archiving and communication system (PACS) environment with multiplanar reformation capabilities (Merge PACS, Merge Healthcare). Each data set was evaluated in multiplanar reformation format aligned to the falx (sagittal plane) and plane of the hippocampus (axial plane). Image review was performed on the coronal oblique plane perpendicular to the hippocampal formations (Fig. 1). All measurements and subjective evaluations of temporal lobe morphology were assessed and agreed on by 2 board-certified radiologists: a pediatric radiology fellow (R.A.) with 5 years' experience evaluating brain MRI and a senior radiologist with a certificate of added qualification in neuroradiology and 22 years' experience evaluating brain MR images in the context of epilepsy surgery (J.L.L.). Each mesial temporal lobe (MTL) in both groups $(n=192)$ was evaluated by an initial rater (R.A.) and subsequently checked by an additional rater (J.L.L.), and any discrepancies were resolved by consensus and were confirmed by measurement criteria described below. Raters were blinded to group, surgical side, and clinical MRI report.

Collateral sulcus angle (CSA) measurements were made with respect to the falx (the smaller the angle, the more vertical the sulcus, Fig. 1C), a modification of techniques used by Barsi et al. ${ }^{4}$ and Okada et al. ${ }^{25}$ As there is variability in the CSA from the anterior to posterior temporal lobe in the oblique coronal plane,,$^{2,4,18}$ we standardized measurement at the most vertical collateral sulcus encountered between the anterior and posterior commissures (Fig. 1D). When the sulcus had a curved morphology, the axis was interpreted as the line joining its cephalad and caudal limits. Both reviewers measured the CSA, and mean values were used for analysis. If there was a greater than $5^{\circ}$ difference between reviewers, a consensus evaluation was performed, and that angle was used. Lateralization of collateral sulcus angle was assessed using 2 

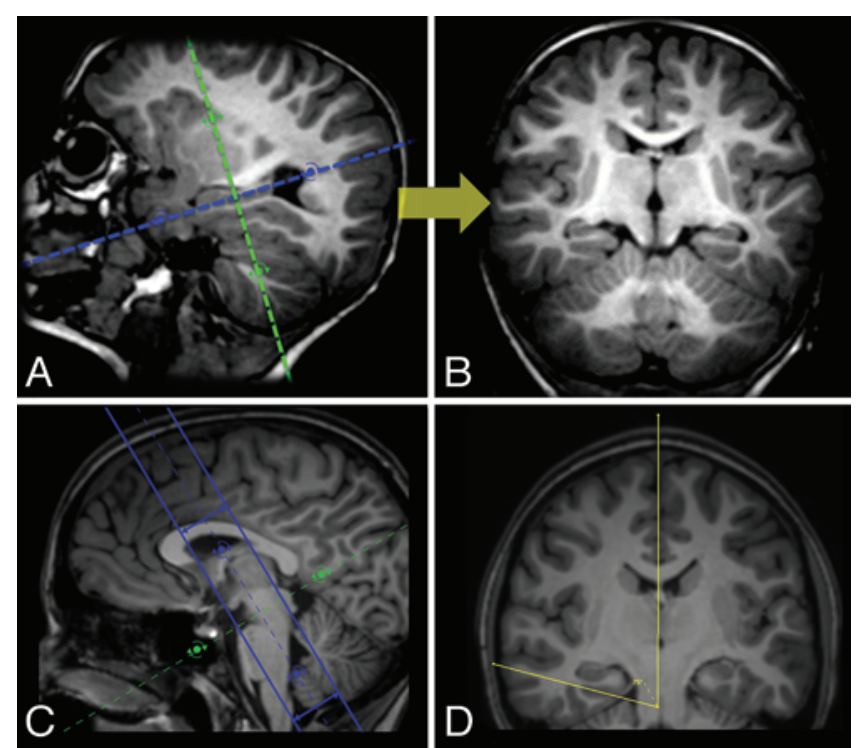

FIG. 1. A: Multiplanar reformation plane perpendicular to the hippocampus chosen in the PACS system for subsequent analysis. B: Reformatted coronal oblique image perpendicular to the hippocampus. C: Region of the hippocampus and MTL evaluated in each case (between the anterior and posterior commissures), typically encompassing the mid to posterior body of the hippocampus (blue lines). D: Definition of the CSA. The angle of the CSA was defined as the smallest angle from perpendicular (falx) of the collateral sulcus between the anterior and posterior commissures (defined in C). A smaller angle represents a more vertical CSA. Figure is available in color online only.

methods: 1) raw CSA lateralization (left < right, left more acute; right $<$ left, right more acute); and 2) CSA lateralization index $(\mathrm{LI})[\mathrm{CSA} \mathrm{LI}=($ left - right $) /($ left + right $)$; left lateralization if $<-0.1$, right lateralization if $>0.1]$. There is little in the literature regarding the normal distribution of the CSA given different techniques and different criteria used. We used $<60^{\circ}$ from vertical as the cutoff in our study, to capture previously reported typical CSAs in HIMAL (CSA $>30^{\circ}$ from horizontal). ${ }^{4}$

Eight specific imaging features were assessed in each MTL. These included the following (Fig. 2): 1) rounded hippocampus (confirmed by documenting equal or greater craniocaudal vs mediolateral dimension) typically medially positioned relative to the medial parahippocampal gyrus; 2) blurring of internal hippocampal architecture; 3) vertical collateral sulcus (confirmed as $<60^{\circ}$ from vertical); 4) asymmetrical wider temporal horn (confirmed as $>1 \mathrm{~mm}$ transverse difference between sides); 5) lower fornix position (confirmed as $>2 \mathrm{~mm}$ difference between sides); 6) asymmetrical wider choroidal fissure (confirmed as $>1 \mathrm{~mm}$ difference between sides); 7) abnormal hippocampal signal; and 8) imaging findings compatible with hippocampal sclerosis (small hippocampus with abnormal signal and ill-defined internal architecture). ${ }^{8,14}$

Findings $1-5$ were used by Barsi et al. ${ }^{4}$ to define HIMAL. They are similar to those initially described by Baulac et al. ${ }^{3}$ and have been evaluated by most studies assessing this imaging anomaly. Findings 6-8 are associated findings either assessed by other authors ${ }^{5}$ or potentially associated conditions. ${ }^{31}$

Assessments of choroidal fissure width, fornix position,
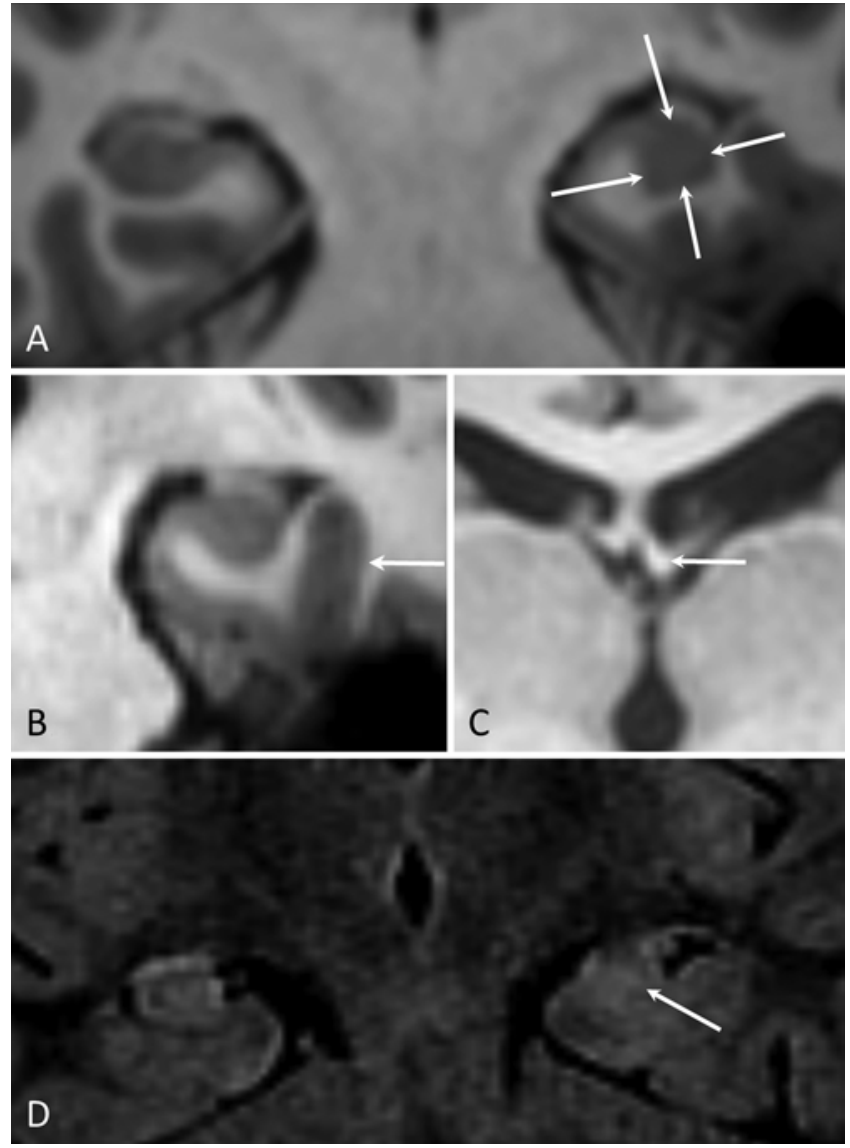

FIG. 2. Examples of evaluated MRI features (volumetric T1-weighted images [A-C], coronal reformatted T2-weighted FLAIR sequence

[D]). A: Rounded left hippocampus (arrows). Note the horizontal position of the right hippocampus and relatively horizontal position of the right collateral sulcus). B: Vertical collateral sulcus (arrow). C: Lower left fornix (arrow). D: Blurred architecture of the left hippocampus.

and temporal horn width were also assessed between the anterior and posterior commissures in the oblique coronal plane perpendicular to the plane of the hippocampus.

Based on prior definitions, ${ }^{2,4,12}$ we based a subjective definition of probable HIMAL on the simultaneous presence of these features: 1) rounded hippocampus; 2) blurring of internal hippocampal architecture; and 3) ipsilateral vertical collateral sulcus. Findings suggestive of HIMAL were the presence of rounded hippocampus and ipsilateral vertical collateral sulcus. This definition in our experience is that which is typically noted clinically by most neuroradiologists as HIMAL. Assessment of blurring was challenging on the 3D T2-weighted FLAIR and T1-weighted imaging sequences used for this study (as they were common to both the seizure and control populations). An MTL score was calculated for each temporal lobe based on the number of these features identified (maximum score of 8).

Surgical outcome was based upon the ILAE outcome grading system (1-6), where 1 is seizure freedom and 6 is $100 \%$ increase in baseline seizure days. ${ }^{36}$ All subjects had at least 1 year of clinical follow-up after surgery. Outcome grades of 1-3 (seizure free to 1-3 seizures/year) were classified as a good surgical outcome. ${ }^{23}$ 
Surgical side, pathological diagnosis, and imaging findings elsewhere in the brain were tabulated. Presence of HIMAL, associated imaging features, and MTL score were compared between sides, between epilepsy and normal groups, in relationship to eventual side of surgery, and in relationship to outcome.

The chi-square or Fisher's exact test was used with categorical and frequency data. The Student t-test (corrected for unequal variance when necessary) was used for continuous and interval variables, and the Wilcoxon rank-sum test or Mann-Whitney U-test was used for ordinal data; $p$ $<0.05$ was used as the cutoff for significance.

\section{Results}

\section{Demographics, Pathology, and Surgery}

No significant group differences in age, sex, or handedness between the seizure and control populations were noted. There were 27 males and 21 females in each group. The mean age in the seizure group was 9.7 years (range 4 months -20 years) and that in the control group was 9.6 years (range 3 months -18 years; $p=0.93$ ). There were 13 left-handed, 33 right-handed, and 2 nondeclared handedness subjects in the seizure group and 4 left-handed, 39 right-handed, and 5 nondeclared handedness subjects in the control group. Although there were more left-handed subjects in the seizure group, this difference was not statistically significant $(p=0.24)$.

For the epilepsy group, imaging findings included findings of cortical dysplasia $(\mathrm{CD})^{22}(\mathrm{n}=20)$, hippocampal sclerosis $(n=5)$, nontemporal lobe encephalomalacia $(n=$ $3)$, and nonlesional/normal $(\mathrm{n}=23)$.

All surgically treated patients had CD identified on histopathology from resected regions. By highest dysplasia grade there were 16 patients with Type I, 18 patients with Type IIa, 7 patients with Type IIb, 2 patients with Type IIIa (hippocampal sclerosis and adjacent Type I CD), and 5 patients with dual pathology (1 each with encephalomalacia and Type IIa CD, hippocampal sclerosis and Type IIa $\mathrm{CD}$, hippocampal sclerosis and Type IIId CD [encephalomalacia and adjacent Type I CD], encephalomalacia and noncontiguous Type I CD, and Type IIa CD and noncontiguous Type I CD). ${ }^{6}$

Resections included anterior temporal lobectomy and amygdalohippocampectomy (ATAH, $n=4)$, ATAH and frontal corticectomy $(n=4)$, ATAH and occipital corticectomy $(\mathrm{n}=1)$, ATAH and parietal corticectomy $(\mathrm{n}=$ $1)$, ATAH and parietal and occipital corticectomy $(n=1)$, ATAH and additional posterior temporal corticectomy (n $=2$ ), frontal corticectomy $(\mathrm{n}=17)$, frontal and parietal corticectomy $(\mathrm{n}=2)$, frontal parietal and occipital corticectomy $(n=1)$, parietal corticectomy $(n=3)$, temporal and parietal corticectomy $(\mathrm{n}=1)$, and functional hemispherotomy $(\mathrm{n}=11)$. There were 26 left-sided surgeries and 22 right-sided surgeries.

\section{Imaging Findings}

Only 1 epilepsy subject $(1 / 48,2 \%)$, and no healthy control exhibited all 5 imaging findings of HIMAL. Imaging findings consistent with probable HIMAL (rounded hippocampus, vertically oriented collateral sulcus, and blur- ring of internal hippocampal architecture) were identified in 3 of $48(6.2 \%)$ surgically treated children with intractable epilepsy and none of the healthy controls, although this did not reach statistical significance $(p=0.12)$. Eight of 48 (16.7\%) epilepsy patients had rounded hippocampus and vertical collateral sulcus (suggesting HIMAL) compared with 2 of 48 (4.6\%) healthy subjects, which did reach statistical significance $(p=0.045)$. No sex differences were noted in the prevalence of any feature.

All evaluated MTL imaging features except lower fornix position and rounded hippocampus were significantly more prevalent in the epilepsy group (Table 1). In healthy subjects, all findings except larger temporal horn were more prevalent on the left; however, only lower fornix position reached statistical significance, side to side $(\mathrm{p}=$ 0.035, Fig. 3).

In seizure patients all MRI findings except larger temporal horn and hippocampal sclerosis were more prevalent on the left (vertical collateral sulcus and lower fornix reached statistical significance, $p=0.035$; Fig. 4). No difference in MTL scores between sides was noted in either group $(\mathrm{z}=-1.46, \mathrm{p}=0.09$ [healthy group], $\mathrm{z}=-1.32$, $\mathrm{p}$ $=0.07$ [seizure group]). Overall, epilepsy subjects had higher MTL scores compared with controls $(\mathrm{z}=-2.95, \mathrm{p}$ $=0.002) ; 14.6 \%$ (7/48) of epilepsy subjects had an MTL score $\geq 3$ compared with $1 \%$ of controls $(\mathrm{p}=0.0003)$. This difference was also present when comparing right and left sides with side-matched controls (MTL score $\geq 3,6 / 48$ seizure vs $0 / 48$ control, right; $9 / 48$ seizure vs $1 / 48$ control, left; $\mathrm{p}=0.013$ and $\mathrm{p}=0.008$, respectively).

In 3 of 5 seizure patients with both a rounded hippocampus and a vertical collateral sulcus, there was an asymmetrically lower fornix. This finding was on the left in all 3 cases. In 2 of 5 cases of hippocampal sclerosis, there was also a rounded hippocampus and vertical collateral sulcus (both on the same side as the HS).

\section{Temporal Versus Extratemporal Resections}

Subjects were dichotomized as undergoing temporal lobe resections only (TLR group, $n=6$, excluding subjects with functional hemispherotomies) and those undergoing extratemporal resections only (ETR group, $\mathrm{n}=23$ ) (Table 2). Rounded hippocampus and vertical collateral sulcus (suggesting HIMAL) were noted in $33 \%$ of patients in the TLR group and 9\% of those in the ETR group versus $4 \%$ of controls. Although there was a higher prevalence of rounded hippocampi and associated vertical collateral sulci in TLR subjects compared with both matched controls and ETR patients, this was not statistically significant, given the small numbers.

Patients undergoing TLR had higher overall MTL scores than controls and those patients with ETR $(p=$ 0.003 and $p=0.049$, respectively). There were also significantly more TLR patients with an MTL score of $>3$ (44\%) than healthy controls $(13 \%), p=0.03$, but not ETR $(p=0.13)$. No significant difference in overall MTL score was noted between those patients with ETR and matched healthy controls. There were, however, more ETR patients with an MTL score of $>3(17 \%)$ than controls $(2.1 \%, \mathrm{p}=$ $0.03)$. 
TABLE 1. Imaging findings in all intractable epilepsy and control subjects

\begin{tabular}{|c|c|c|c|c|c|c|c|c|c|}
\hline \multirow[b]{2}{*}{ Finding } & \multicolumn{3}{|c|}{ Control $(n=48)$} & \multicolumn{3}{|c|}{ Seizure $(n=48)$} & \multicolumn{3}{|c|}{ Group Comparisons ( $p$ value) } \\
\hline & Right & Left & Total & Right & Left & Total & Total & Right & Left \\
\hline Rounded & 1 & 3 & 4 & 4 & 7 & $10 \dagger$ & $0.07 \ddagger$ & 0.18 & 0.16 \\
\hline Blurring & 0 & 0 & 0 & 3 & 7 & $9 \S$ & 0.001 & 0.12 & 0.006 \\
\hline Vertical CS & 0 & 4 & 4 & 3 & 10 & $12 ף$ & 0.026 & 0.12 & 0.07 \\
\hline Rounded HC \& vertical CS & 0 & 2 & 2 & 3 & 6 & $8^{* *}$ & 0.045 & 0.12 & 0.13 \\
\hline Wider choroidal fissure & 0 & 2 & 2 & 6 & 6 & 12 & 0.037 & 0.01 & 0.13 \\
\hline Larger temporal horn & 5 & 2 & 7 & 12 & 9 & 21 & 0.002 & 0.05 & 0.025 \\
\hline Lower fornix & 3 & 10 & 13 & 3 & 10 & 13 & 0.6 & 0.66 & 0.6 \\
\hline Abnormal signal & 0 & 0 & 0 & 3 & 5 & $7 \dagger \dagger$ & 0.006 & 0.12 & 0.03 \\
\hline Hippocampal sclerosis & 0 & 0 & 0 & 3 & 3 & 6 & 0.01 & 0.12 & 0.12 \\
\hline
\end{tabular}

$\mathrm{CS}=$ collateral sulcus; $\mathrm{HC}=$ hippocampus.

* The Fisher exact test was used for group comparisons. Boldface type indicates statistical significance.

$\dagger$ One patient had bilateral rounded hippocampi.

$\ddagger$ Although a rounded hippocampus was more prevalent in the epilepsy group $(20.8 \%$ vs $8.3 \%)$, this did not reach statistical significance $(p=$

$0.07)$.

§ One patient had bilateral internal hippocampal architecture blurring.

II One patient had bilateral vertical collateral sulci.

** One patient had bilateral rounded hippocampi and vertical collateral sulci.

†† One patient had bilateral abnormal hippocampal signal.

\section{Collateral Sulcus Angle}

The left CSA was more acute (mean $67.3^{\circ}$ [control group], $63.9^{\circ}$ [epilepsy group]) than the right (mean $74.7^{\circ}$ [control group], $71.3^{\circ}$ [epilepsy group] $(\mathrm{p}<0.0001$; Fig. $5)$. Both CSAs were significantly more acute in epilepsy subjects, compared with side-matched control subjects ( $\mathrm{p}$ $=0.04$, left $; \mathrm{p}=0.01$, right).

\section{Correlation With Surgical Side}

There was no significant association with surgical side in those with round hippocampi and vertical collateral sulci or lateralizing CSAs (Table 3). Lateralizing raw MTL scores did have a significant association with surgical side $(\mathrm{p}=0.03$, OR 7.33), although this was not persistent when hippocampal sclerosis cases were excluded (see below). No significant correlation was found between any finding in isolation and surgical side (data not shown).

\section{Outcome Assessment}

Twenty-seven patients (56\%) had a good surgical out-

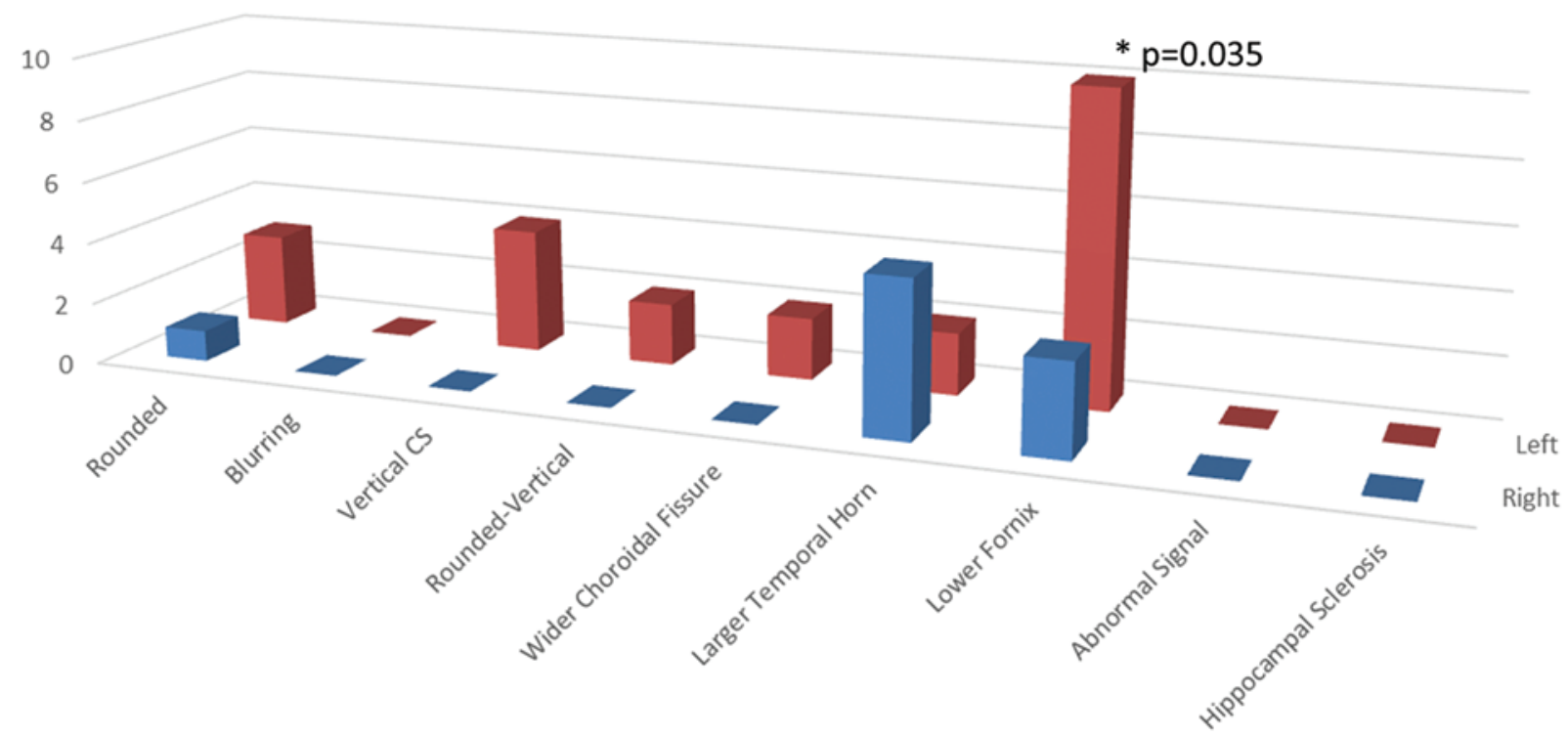

FIG. 3. Imaging features in healthy subjects comparing left and right hemispheres ("statistically significant). The lower fornix was more prevalent on the left $(p=0.035)$. The larger temporal horn was more prevalent on the right but was not statistically significant. All other features, when present, were more prevalent on the left but were not statistically significant. No control subjects exhibited hippocampal blurring, increased hippocampal signal, or findings of hippocampal sclerosis. CS = collateral sulcus. Figure is available in color online only. 


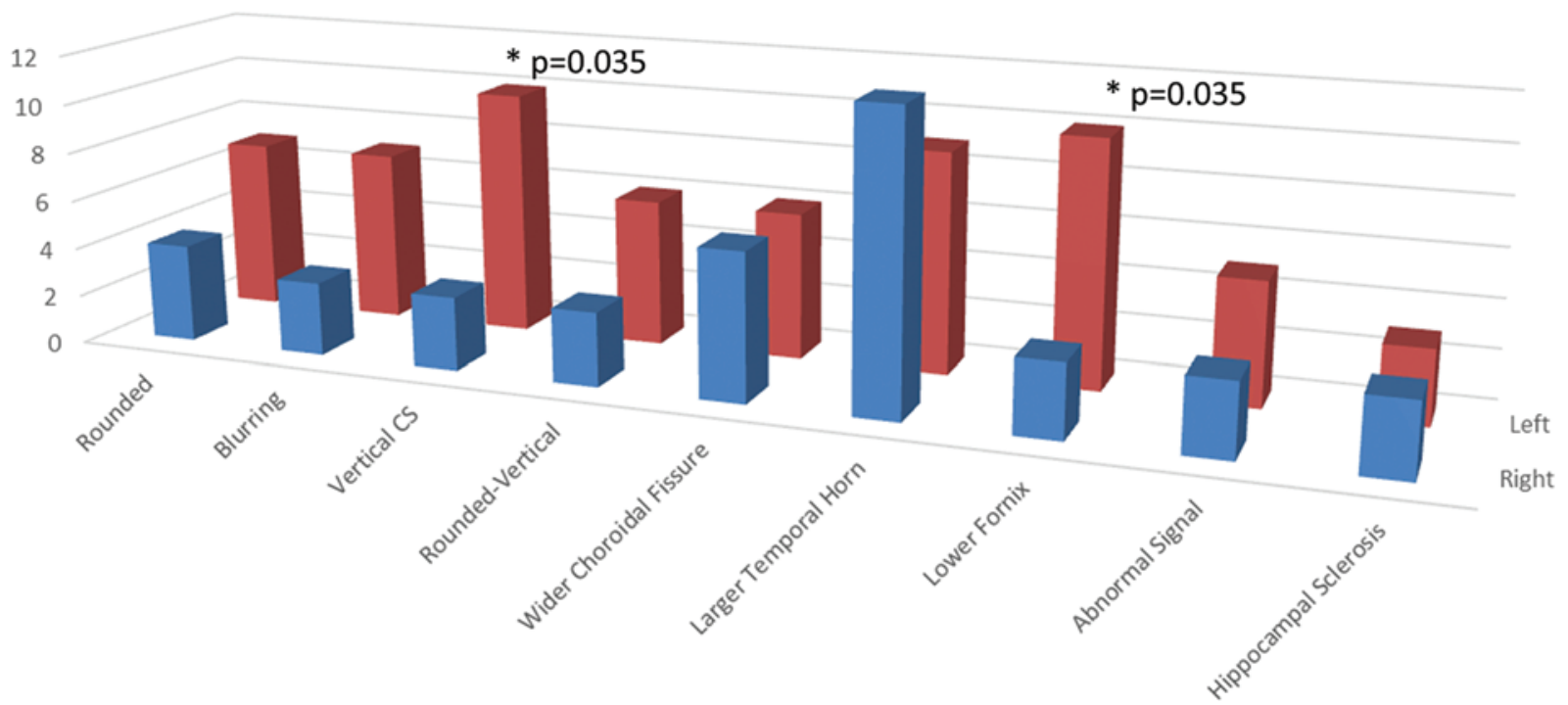

FIG. 4. Imaging features in intractable epilepsy patients comparing left and right hemispheres ("statistically significant). All features except larger temporal horn hippocampal sclerosis were more prevalent on the left, lower fornix position and vertical collateral sulcus, reaching statistical significance $(p=0.035)$. Figure is available in color online only.

come (ILAE outcome grade of 1-3). No significant difference in outcome was noted between those subjects with a rounded hippocampus and vertical collateral sulcus, or rounded hippocampus, vertical collateral sulcus, and architectural blurring and those without these findings ( $\mathrm{p}$ $=0.22$ and $\mathrm{p}=0.6$, respectively). Of the 8 patients with rounded hippocampi and associated vertical collateral sulci, 4 had a malrotated hippocampus resected (1 had bilateral hippocampal findings with a remaining malrotated hippocampus and 3 had only ETRs). Two of these patients had imaging and pathological evidence of hippocampal sclerosis. Of the patients with a persistent malrotated hippocampus remaining after surgery, 2 of 4 had good outcome $(50 \%)$ compared with 4 of $4(100 \%)$ of the patients with the abnormal hippocampus resected. Given the small numbers, this was not statistically significant.

No significant difference was noted in prevalence of any MTL finding in those with good versus poor outcomes (data not shown). There was a significant correlation of total MTL score (combined score of both temporal lobes) and outcome $(\mathrm{z}=3.67, \mathrm{p}=0.002)$. And $81 \%(22 / 27)$ of patients with good outcome had an MTL score of at least 1 versus $48 \%(10 / 21)$ patients with a poor outcome $(p=0.03)$. No significant difference in outcome was noted comparing those patients with left- or right-sided resections and leftor right-sided MTL score, respectively.

\section{Analysis With Hippocampal Sclerosis Cases Removed}

To better understand the relationship of hippocampal sclerosis to HIMAL and outcome, an analysis was performed with those patients removed. When hippocampal sclerosis subjects were excluded, only a wide choroidal fissure and asymmetrically larger temporal horn were significantly more prevalent in seizure patients compared with controls (Table 4). The combination of rounded hippocampus and vertical collateral sulcus (suggesting HIMAL) was more prevalent in epilepsy subjects $(6 / 43 ; 14 \%)$ compared with controls $(2 / 43 ; 4.6 \%)$, but did not reach statistical significance $(\mathrm{p}=0.13)$.

Excluding hippocampal sclerosis patients, seizure patients still had higher MTL scores compared with controls ( $\mathrm{z}=-2.45, \mathrm{p}=0.007$ ), although lateralizing raw MTL score did not have a significant association with surgical side. Significantly more patients with intractable seizures $(10.5 \%)$ had an MTL score of 3 or greater than controls $(1 \%)(p=0.0009)$, although this difference did not reach statistical significance when side matched. Even when

TABLE 2. Comparison of patients who underwent TLR versus those who underwent ETR

\begin{tabular}{|c|c|c|c|c|c|c|}
\hline \multirow[b]{2}{*}{ Feature } & \multirow[b]{2}{*}{$\operatorname{TLR}(n=6)$} & \multirow[b]{2}{*}{$\operatorname{ETR}(n=23)$} & \multirow[b]{2}{*}{ Control $(n=48)$} & \multicolumn{3}{|c|}{ Group Comparisons } \\
\hline & & & & TLR vs Control & ETR vs Control & TLR vs ETR \\
\hline Rounded HC \& vertical CS & $2(\mathrm{It} ; 33 \%)$ & 2 (It; 9\%) & $2(\mathrm{lt} ; 4.2 \%)$ & $p=0.056$ & $p=0.59$ & $p=0.18$ \\
\hline MTL score (combined) & & & & $z=2.3 ; p=0.02$ & $z=0.9 ; p=0.37$ & $z=1.97 ; p=0.049$ \\
\hline Median & 2.5 & 0 & 0 & & & \\
\hline Range & $0-8$ & $0-3$ & $0-3$ & & & \\
\hline Mean & 3.3 & 1.1 & 0.56 & & & \\
\hline MTL score >3 (combined) & $3(50 \%)$ & $4(17.4 \%)$ & $1(2.1 \%)$ & $p=0.003$ & $p=0.03$ & $p=0.13$ \\
\hline
\end{tabular}

* Boldface type indicates statistical significance. 


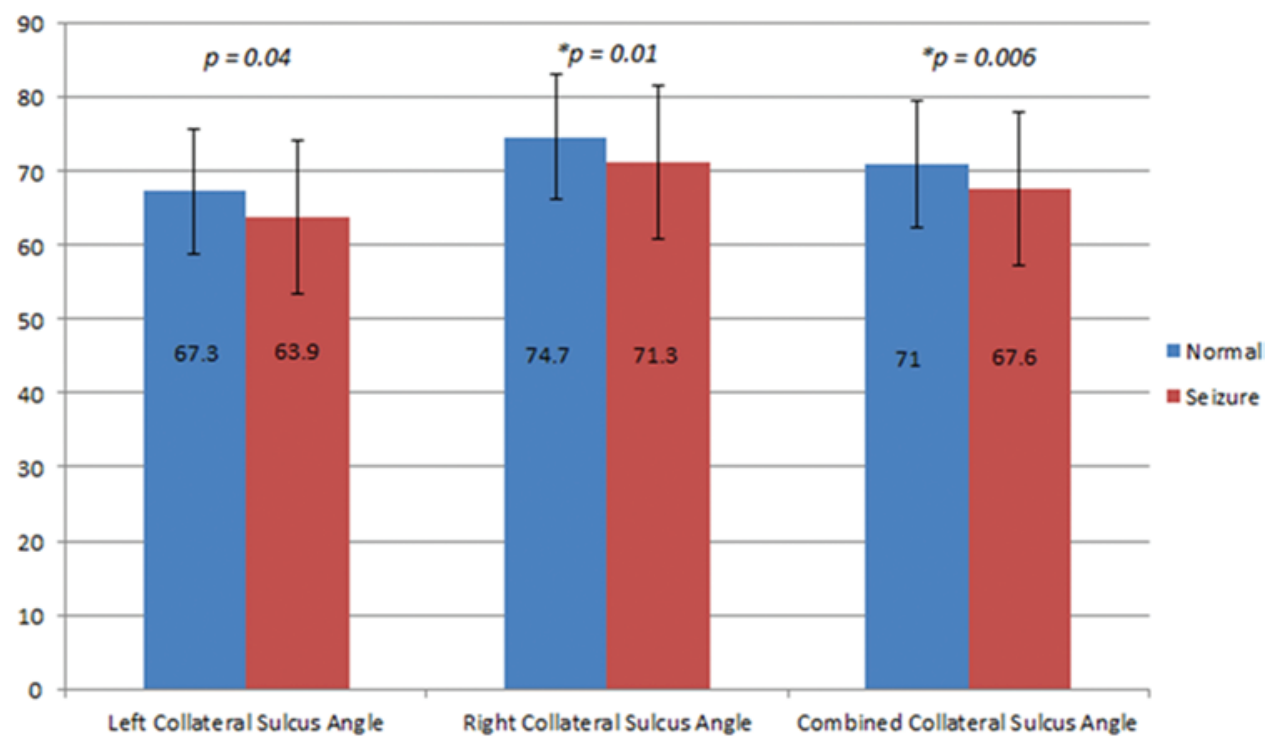

FIG. 5. Collateral sulcus angles (in degrees) in normal controls and intractable epilepsy patients (*statistically significant). Significant differences persisted when combining right and left CSAs $(n=96)$ and comparing normal and epilepsy patients $(p=0.0006)$. Figure is available in color online only.

removing patients with HS, there was a significant correlation of the total MTL score (combined score of both temporal lobes) and outcome with 79\% (19/24) of patients with good outcome having an MTL score of at least 1 versus $42 \%(8 / 19)$ of patients with a poor outcome $(\mathrm{p}=0.02)$.

\section{Pathology of HIMAL}

Of the 4 patients in which a malrotated hippocampus was resected, 2 had hippocampal sclerosis (hippocampal gliosis and neuronal loss in the cornu ammonis 1 (CA1) and CA2 regions of Ammon's horn) with no evidence of frank dysplasia within the hippocampus (one had adjacent Type I CD in the anterior temporal lobe and the other had Type IIa dysplasia in the noncontiguous temporal operculum). The other 2 patients had dysmorphic neurons within

TABLE 3. Surgical side and CSA and round hippocampi and vertical collateral sulci (round/vertical) lateralization

\begin{tabular}{cccc}
\hline \multirow{2}{*}{ Evaluation } & \multicolumn{2}{c}{ Surgical Side } & \\
\cline { 2 - 3 } & Left & Right & p Value \\
\hline CSA (raw angle) & & & 0.74 \\
\hline Lt $<$ rt (It more vertical) & 20 & 15 & \\
\hline Rt < It (rt more vertical) & 6 & 6 & \\
\hline CSA (LI) & Left & Right & 0.82 \\
\hline Lt & 6 & 7 & \\
\hline Rt & 2 & 2 & \\
\hline Nonlateralizing & 18 & 13 & \\
\hline Rounded HC \& vertical CS & Left & Right & 0.64 \\
\hline Lt & 4 & 2 & \\
\hline Rt & 0 & 1 & \\
\hline Bilat & 1 & 0 & \\
\hline
\end{tabular}

$\mathrm{LI}=$ lateralization index (as described in Methods).

* In one subject, the CSA was the same on both sides (raw angle was not lateralizing). the hippocampus (one with dysmorphic neurons in the CA3 and CA4 regions and the other with dysmorphic neurons in the CA4 region, both consistent with Type IIa CD in the hippocampus).

\section{Discussion}

Our evaluation of temporal lobe morphology in 48 children with intractable epilepsy undergoing resection (compared with sex-, age-, and handedness-matched healthy children) adds to the literature concerning MTL morphology and seizures and helps define the significance of the finding typically known as HIMAL in this cohort. The full complement of imaging features of HIMAL was rarely seen. Only one epilepsy patient $(1 / 48 ; 2 \%)$ and no control subject exhibited all the typically described imaging findings of HIMAL. Using less strict criteria (only the combination of rounded hippocampus and vertical collateral sulcus), HIMAL-type morphology occurs more commonly in surgically treated pediatric epilepsy patients $(16.7 \%)$ versus controls $(4.6 \% ; \mathrm{p}=0.045)$; however, this was not significant when patients with hippocampal sclerosis were removed from analysis. HIMAL and related findings were more prevalent in subjects with TLRs versus those with only ETRs, but this did not predict side of eventual surgery and was not significantly associated with outcome. Pathologically, hippocampal sclerosis and/or dysplastic changes were seen in the small number of patients in which the malrotated hippocampus was resected. Outcome was good in all 4 of the patients who underwent removal of the malrotated hippocampus, although this was not statistically significant compared with those patients in which the malrotated hippocampus remained after surgery.

\section{Developmental Factors}

Recent studies have suggested that alterations in temporal lobe morphology compatible with HIMAL occur 
TABLE 4. Imaging findings in intractable epilepsy and control subjects (5 hippocampal sclerosis cases removed)

\begin{tabular}{|c|c|c|c|c|c|c|c|c|c|}
\hline \multirow[b]{2}{*}{ Finding } & \multicolumn{3}{|c|}{ Control $(n=43)$} & \multicolumn{3}{|c|}{ Seizure $(n=43)$} & \multicolumn{3}{|c|}{ Group Comparisons ( $p$ value) } \\
\hline & Right & Left & Total & Right & Left & Total & Total & Right & Left \\
\hline Rounded & 1 & 2 & 3 & 3 & 6 & $8 \dagger$ & 0.1 & 0.3 & 0.13 \\
\hline Blurring & 0 & 0 & 0 & 1 & 3 & 4 & 0.06 & 0.5 & 0.12 \\
\hline Vertical CS & 0 & 4 & 4 & 2 & 9 & $10 \ddagger$ & 0.07 & 0.25 & 0.11 \\
\hline Rounded HC \& vertical CS & 0 & 2 & 2 & 2 & 5 & $6 \S$ & 0.13 & 0.25 & 0.21 \\
\hline Wider choroidal fissure & 0 & 1 & 1 & 4 & 5 & 9 & 0.007 & 0.06 & 0.1 \\
\hline Larger temporal horn & 5 & 1 & 6 & 10 & 8 & 18 & 0.004 & 0.13 & 0.015 \\
\hline Lower fornix & 3 & 9 & 12 & 3 & 8 & 11 & 0.5 & 0.67 & 0.5 \\
\hline Abnormal signal & 0 & 0 & 0 & 0 & 1 & 1 & 0.5 & NA & 0.5 \\
\hline Hippocampal sclerosis & 0 & 0 & 0 & 0 & 0 & 0 & NA & NA & NA \\
\hline
\end{tabular}

NA = not applicable.

* The Fisher exact test was used for group comparisons. Boldface type indicates statistical significance.

$\dagger$ One patient had bilateral rounded hippocampi.

$\ddagger$ One patient had bilateral vertical collateral sulci.

$\S$ One patient had bilateral rounded hippocampi and vertical collateral sulci.

in a higher prevalence in patients with epilepsy and may indicate a developmental alteration that may predispose patients to febrile status epilepticus and potentially the development of chronic epilepsy. ${ }^{1,5,12,31}$ The exact definition of HIMAL has been debated, and the inclusion of multiple, potentially related findings has not been accepted by all researchers. ${ }^{1,12}$ The association between HIMAL and febrile status has also recently been questioned. ${ }^{9}$ Even the term "hippocampal malrotation" has been debated as an appropriate term with some authors suggesting that "incomplete hippocampal inversion" is a more embryologically appropriate description. ${ }^{27}$

It has been postulated that disruption of the normal process of hippocampal infolding results in the imaging features of HIMAL. The hippocampal formation is one of the first cortical areas to differentiate, and the main components of the hippocampus progressively grow, change shape, and alter their relationship to each other and adjacent gyri, resembling its adult morphology by Week $17-18 .{ }^{13}$ The hippocampal fissure progressively changes from vertical to transverse, becoming similar to the adult pattern by the 30th week..$^{17}$ Fetal MRI studies document that the hippocampal infolding angle continues to become more horizontal throughout late fetal development (20-37 weeks). ${ }^{28}$ Postnatal imaging studies have documented continued changes in hippocampal volume throughout childhood with a right-left asymmetry (right being larger). ${ }^{33}$ It has been postulated that the right hippocampus starts developing earlier or develops faster. ${ }^{2,25}$ If a process stops the infolding, then inversion could be completed on the right side but not on the left. ${ }^{2}$ Interestingly, there is a higher prevalence of bilateral HIMAL in patients with significant gross developmental anomalies (especially callosal agenesis, lissencephaly, and periventricular nodular heterotopias)..$^{11,21,24,29}$ Bilateral HIMAL is quite rare in epilepsy populations without extensive midline or significant migration/organizational abnormalities or healthy/control populations without epilepsy. These findings support that HIMAL is primarily developmental in etiology.

\section{Prevalence of HIMAL and Side-Side Differences}

The prevalence of probable HIMAL (6\%) and findings suggesting HIMAL (16.7\%) in our epilepsy cohort is similar to that found in other studies, which have been primarily in adults. Barsi et al. ${ }^{4}$ found HIMAL (strictly defined) in $28(5 \%)$ of 527 patients who underwent imaging because of suspicion of epilepsy, similar to the $6 \%$ identified in our series. Bernasconi et al., ${ }^{5}$ in a group of patients with intractable temporal lobe epilepsy, identified rounded, vertically oriented hippocampi in $27 \%$ and vertical collateral sulci in $23 \%$, similar to the $20 \%$ and $25 \%$ prevalence of these findings in our study. Bajic et al. ${ }^{1}$ found "incomplete hippocampal inversion" (essentially a rounded medialized hippocampus and vertical collateral sulcus) in a larger percentage of epilepsy patients (30\%) than that found in other reports and our study. Interestingly, they found the highest prevalence in cryptogenic generalized (57\%), idiopathic rolandic (44\%), and cryptogenic focal (80\%) epilepsy syndromes. When evaluating symptomatic focal epilepsies (analogous to our cohort) they identified a prevalence of $21 \%$, more similar to our study. Kuchukhidze et al., ${ }^{21}$ in a study of hippocampal morphology in a primarily adult population with malformations of cortical development and epilepsy, found HIMAL in approximately $13 \%$ of 59 patients with CD similar to the prevalence of rounded hippocampus and vertical collateral sulcus in our pediatric surgical population with $\mathrm{CD}$.

Gamss et al., in a large primarily adult (youngest subject was 15 years old) clinical nonseizure cohort, did not identify strictly defined HIMAL (including all features described in this study) in any of their 497 subjects. ${ }^{12} \mathrm{~A}$ rounded hippocampus and vertical collateral sulcus occurred in 6 of 497 subjects (1.2\%), slightly smaller than the prevalence in our control cohort. In the few healthy control populations evaluated (similar to our control population), the prevalence of rounded hippocampi has been seen in $12 \%,,^{5} 8 \%,{ }^{10}$ and $3 \%$ of subjects, similar to our normal cohort prevalence. 
Overall, our study supports that strictly defined HIMAL is rarely seen in normal subjects and is more commonly identified in patients with epilepsy. Isolated rounded configuration of the hippocampus with or without associated vertical collateral sulcus configuration is rarely but more commonly seen in controls and not uncommonly identified in patients with epilepsy. Our study defines this prevalence for the first time in a surgically treated pediatric epilepsy population.

Findings in our study and others demonstrate that many features of HIMAL, including rounded hippocampi, vertical collateral sulcus, and more inferior fornix position, are more prevalent in the left hemisphere in both healthy children and those with intractable epilepsy.,2,4,10,12,31 This suggests that there are normal developmental differences between left and right temporal lobes, which may become accentuated in epilepsy patients. Our findings of left lateralization of a rounded hippocampus in $67 \%$, vertical collateral sulcus in $82 \%$, and rounded hippocampus and vertical collateral sulcus in $71 \%$ of intractable epilepsy patients are consistent with those in these prior reports. In addition, we found that the collateral sulcus, closely related to hippocampal development, is more acute on the left compared with the right in both our epilepsy and healthy control populations, also similar to prior studies.

These findings are not surprising as asymmetries in MTL structures have been repeatedly described in control and epilepsy populations both quantitatively and qualitatively. Gamss et al. found a rounded hippocampus and vertical collateral sulcus in 6 of 497 patients without epilepsy (all on the left side). ${ }^{12}$ Similarly, Bajic et al. found findings consistent with incomplete hippocampal inversion in 19 of 100 nonepilepsy subjects, also all on the left side. ${ }^{2}$ This leftward predominance of rounded hippocampal morphology was also noted by Connor et al. in schizophrenic and healthy control populations, ${ }^{10}$ where $72 \%$ of rounded hippocampi were left sided. Leftward predominance of HIMAL findings has also been described in most epilepsy cohorts $\left(67 \%^{1}\right.$ to $\left.69 \%{ }^{3,4}\right)$. Shinnar et al. described left-sided lateralization of HIMAL in $87 \%$ when seen in patients presenting with febrile status epilepticus. ${ }^{31}$ Okada et al. ${ }^{25}$ demonstrated a more vertical hippocampal angle (which correlates with CSA) on the left in a mixed healthy and epilepsy population.

The right hippocampus, as well as the temporal lobe, is typically larger throughout postnatal development and in adults..$^{14,26,32,33}$ Differential development between the right and left hippocampi likely occurs in humans, explaining the right/left asymmetry in findings of HIMAL. Based on the findings in our study, in concert with other investigations, findings of HIMAL in the right temporal lobe may be more likely to reflect abnormal brain development and harbor potential pathological significance.

We found that the collateral sulcus angle is more acute (even when comparing matched sides) and that MTL scores (sum of temporal lobe findings associated with HIMAL) are larger in epilepsy patients than in control subjects. This finding was noted even when hippocampal sclerosis cases (which would add to the score given internal architecture blurring and increased signal as a definition) were removed from analysis. This suggests that while there are develop- mental asymmetries in healthy subjects, these are accentuated in patients with intractable epilepsy.

\section{Value for Presurgical Assessment}

Our study is the first to our knowledge to assess HIMAL as a lateralizing finding in pediatric intractable epilepsy surgery. A finding of a more acute CSA, rounded hippocampus, and vertical collateral sulcus, or other associated MTL findings did not correlate with eventual surgical side and thus cannot be used for presurgical lateralization in this population. Only raw MTL score lateralized to the surgical side, probably related to the presence of hippocampal sclerosis, as this statistically significant association was not present when these cases were removed from analysis. Based on prior studies that demonstrated poor correlation of EEG laterality and HIMAL, this was not unexpected. 3,5

We could find no other study assessing the difference of prevalence of HIMAL and associated findings comparing TLRs and ETRs in an epilepsy surgery cohort. We found a higher prevalence of HIMAL (rounded hippocampus and vertical collateral sulcus) in those patients who underwent TLR (33\%) versus those who underwent ETR (9\%) and control subjects (4\%), although given small numbers, this was not statistically significant. A higher prevalence of associated findings (higher MTL score) was noted in patients with TLR versus patients with ETR as well as healthy controls. These findings suggest that patients with temporal lobe seizures prompting TLR have an increased prevalence of structural developmental changes in the temporal lobes compared with those patients with extratemporal localization. This finding correlates with prior studies demonstrating altered cortical folding complexity in the temporal lobes and HIMAL in patients with temporal lobe epilepsy. ${ }^{18,35}$ While not necessarily lateralizing, some findings of HIMAL may have a lobar localizing value. This may have important implications for presurgical evaluation, including invasive EEG electrode placement. Our sample was too small to determine whether HIMAL findings contralateral to the resected temporal lobe signify a poor prognosis; however, this would be important to evaluate in a future study with larger numbers of patients with TLRs.

As with other studies, ${ }^{12}$ we found low forniceal position to be common in both normal and seizure subjects and more prevalent on the left in each group. Our findings, in combination with those from other studies, suggest that lower fornix position is most likely an unrelated anatomical variation and should not be used as a criterion for HIMAL. Its significance is uncertain.

We could find no other study that has assessed the neuropathology of HIMAL. In 2 of our 4 patients with resected malrotated hippocampi, findings of hippocampal dysplasia were present. In the other 2 patients, hippocampal sclerosis was present in concert with dysplastic changes in the anterior temporal lobe or temporal operculum. These findings suggest that a malrotated hippocampus may exhibit intrinsic dysplastic changes or be associated with regional neocortical dysplastic findings on histology. This association should be assessed in further studies. 


\section{Limitations}

There are several limitations to the present study. This study is retrospective, assessing a cohort of selected patients for surgical therapy. While control cases were selected to match the epilepsy surgery group demographically, there were slight differences between groups in terms of handedness (while not statistically significant on a population basis), which could potentially still influence temporal lobe morphology. The study evaluated temporal lobe morphology on a subjective visual basis, although this most accurately reflects clinical practice. Subjective findings of vertical CSA, asymmetrical temporal horn width, asymmetrical low fornix position, and rounded hippocampus were subsequently confirmed by measurement, and all findings were agreed upon by consensus opinions of the 2 reviewers. The 2 reviewers, while blinded to pathology and surgical side, did have different levels of experience, and this could have produced bias in the consensus reading. To increase consistency, the CSA, fornix position, temporal horn width, and rounded hippocampal morphology were evaluated in a zone between the anterior and posterior commissures, perpendicular to the hippocampus. Given the variability of the $\mathrm{CSA}^{2,4,18}$ and the potential pitfall of calling a rounded hippocampus because of a scan angle not perpendicular to the hippocampus, these constraints were necessary. Fortunately, HIMAL and associated findings are most prevalent in the body and tail of the hippocampus ${ }^{5,10}$ and would be covered for the most part by this assessment zone. These technical factors should be kept in mind when applying these findings to other clinical groups and comparing with prior studies. CSA measurements, while significantly different among groups and between sides, likely have low clinical utility in individual patients given the relative wide distribution of measurements. Automated, volumetric, or other quantitative approaches may show more subtle differences in temporal lobe morphology that could have lateralizing utility and are a target for further research. . $^{19,35}$

We did not perform a detailed assessment of EEG localization and HIMAL findings. Our goal was to assess the relationship of HIMAL findings to eventual surgical lateralization in children with intractable epilepsy. Given the relatively small numbers of patients and controls, when dichotomizing the subjects by relationship to each side and resection location (TLR vs ETR), important differences may not be statistically significant. Our patient cohort included a high percentage of MRI-negative cases and ETRs. This may produce a selection bias toward patients with extratemporal epilepsy. Conversely, however, this may be a strength as the study is not biased toward TLR, and it may better reflect the overall population of children with intractable epilepsy.

\section{Conclusions}

Findings compatible with HIMAL occur more commonly in children with intractable epilepsy undergoing surgical treatment (2\%-17\%, depending upon definition) than a matched control population $(0 \%-5 \%)$. Many features associated previously with HIMAL are more prevalent on the left in both seizure patients and healthy chil- dren, likely reflecting differing developmental trajectories of each temporal lobe. While increased in prevalence in intractable seizure patients, HIMAL did not have preoperative lateralizing utility in our cohort. As a group, imaging findings of HIMAL (round hippocampus, round hippocampus and vertical collateral sulcus) did not predict outcome after surgery, although our numbers are small and the patient population comprised a large percentage of ETRs. Although not a predictive preoperative lateralizing finding in our study, HIMAL was more common in patients who underwent TLRs, and the small number of patients in which the malrotated hippocampus was removed had good outcomes.

\section{Acknowledgments}

Data presented in this work were obtained from the database known as Cincinnati MR Imaging of NeuroDevelopment (C-MIND), provided by the Pediatric Functional Neuroimaging Research Network at https://research.cchmc.org/c-mind/. This network and the resulting C-MIND database were supported by contract from the Eunice Kennedy Shriver National Institute of Child Health and Human Development (HHSN275200900018C).

\section{References}

1. Bajic D, Kumlien E, Mattsson P, Lundberg S, Wang C, Raininko R: Incomplete hippocampal inversion-is there a relation to epilepsy? Eur Radiol 19:2544-2550, 2009

2. Bajic D, Wang C, Kumlien E, Mattsson P, Lundberg S, EegOlofsson O, et al: Incomplete inversion of the hippocampusa common developmental anomaly. Eur Radiol 18:138-142, 2008

3. Baulac M, De Grissac N, Hasboun D, Oppenheim C, Adam C, Arzimanoglou A, et al: Hippocampal developmental changes in patients with partial epilepsy: magnetic resonance imaging and clinical aspects. Ann Neurol 44:223-233, 1998

4. Barsi P, Kenéz J, Solymosi D, Kulin A, Halász P, Rásonyi G, et al: Hippocampal malrotation with normal corpus callosum: a new entity? Neuroradiology 42:339-345, 2000

5. Bernasconi N, Kinay D, Andermann F, Antel S, Bernasconi A: Analysis of shape and positioning of the hippocampal formation: an MRI study in patients with partial epilepsy and healthy controls. Brain 128:2442-2452, 2005

6. Blümcke I, Thom M, Aronica E, Armstrong DD, Vinters HV, Palmini A, et al: The clinicopathologic spectrum of focal cortical dysplasias: a consensus classification proposed by an ad hoc Task Force of the ILAE Diagnostic Methods Commission. Epilepsia 52:158-174, 2011

7. Bronen RA, Cheung G: MRI of the temporal lobe: normal variations, with special reference toward epilepsy. Magn Reson Imaging 9:501-507, 1991

8. Bronen RA, Cheung G, Charles JT, Kim JH, Spencer DD, Spencer SS, et al: Imaging findings in hippocampal sclerosis: correlation with pathology. AJNR Am J Neuroradiol 12:933-940, 1991

9. Chin R, Pujar S, Krishnan A, Chong K, Neville B, Scott R: Prevalence of hippocampal malrotation (HIMAL) is not higher in children with prolonged febrile seizures compared to age-matched controls: A population based study. Epilepsy Curr 14 Suppl:373-374, 2014 (Abstract)

10. Connor SE, Ng V, McDonald C, Schulze K, Morgan K, Dazzan P, et al: A study of hippocampal shape anomaly in schizophrenia and in families multiply affected by schizophrenia or bipolar disorder. Neuroradiology 46:523-534, 2004

11. Donmez FY, Yildirim M, Erkek N, Demir Karacan C, Coskun M: Hippocampal abnormalities associated with vari- 
ous congenital malformations. Childs Nerv Syst 25:933939, 2009

12. Gamss RP, Slasky SE, Bello JA, Miller TS, Shinnar S: Prevalence of hippocampal malrotation in a population without seizures. AJNR Am J Neuroradiol 30:1571-1573, 2009

13. Humphrey T: The development of the human hippocampal fissure. J Anat 101:655-676, 1967

14. Jack CR Jr, Rydberg CH, Krecke KN, Trenerry MR, Parisi JE, Rydberg JN, et al: Mesial temporal sclerosis: diagnosis with fluid-attenuated inversion-recovery versus spin-echo MR imaging. Radiology 199:367-373, 1996

15. Jack CR Jr, Twomey CK, Zinsmeister AR, Sharbrough FW, Petersen RC, Cascino GD: Anterior temporal lobes and hippocampal formations: normative volumetric measurements from MR images in young adults. Radiology 172:549-554, 1989

16. Kaiser D, Leach J, Vannest J, Schapiro M, Holland S: Unanticipated findings in pediatric neuroimaging research: prevalence of abnormalities and process for reporting and clinical follow-up. Brain Imaging Behav 9:32-42, 2015

17. Kier EL, Kim JH, Fulbright RK, Bronen RA: Embryology of the human fetal hippocampus: MR imaging, anatomy, and histology. AJNR Am J Neuroradiol 18:525-532, 1997

18. Kim H, Bernasconi N, Bernhardt B, Colliot O, Bernasconi A: Basal temporal sulcal morphology in healthy controls and patients with temporal lobe epilepsy. Neurology 70:2159-2165, 2008

19. Kim H, Chupin M, Colliot O, Bernhardt BC, Bernasconi N, Bernasconi A: Automatic hippocampal segmentation in temporal lobe epilepsy: impact of developmental abnormalities. Neuroimage 59:3178-3186, 2012

20. Kim H, Mansi T, Bernasconi N: Disentangling hippocampal shape anomalies in epilepsy. Front Neurol 4:131, 2013

21. Kuchukhidze G, Koppelstaetter F, Unterberger I, Dobesberger J, Walser G, Zamarian L, et al: Hippocampal abnormalities in malformations of cortical development: MRI study. Neurology 74:1575-1582, 2010

22. Leach JL, Greiner HM, Miles L, Mangano FT: Imaging spectrum of cortical dysplasia in children. Semin Roentgenol 49:99-111, 2014

23. Leach JL, Miles L, Henkel DM, Greiner HM, Kukreja MK, Holland KD, et al: Magnetic resonance imaging abnormalities in the resection region correlate with histopathological type, gliosis extent, and postoperative outcome in pediatric cortical dysplasia. J Neurosurg Pediatr 14:68-80, 2014

24. Montenegro MA, Kinay D, Cendes F, Bernasconi A, Bernasconi N, Coan AC, et al: Patterns of hippocampal abnormalities in malformations of cortical development. J Neurol Neurosurg Psychiatry 77:367-371, 2006

25. Okada Y, Kato T, Iwai K, Iwasaki N, Ohto T, Matsui A: Evaluation of hippocampal infolding using magnetic resonance imaging. Neuroreport 14:1405-1409, 2003

26. Pedraza O, Bowers D, Gilmore R: Asymmetry of the hippocampus and amygdala in MRI volumetric measurements of normal adults. J Int Neuropsychol Soc 10:664-678, 2004

27. Raininko R, Bajic D: "Hippocampal malrotation": no real malrotation and not rare. AJNR Am J Neuroradiol 31:E39_ E40, 2010 (Letter)
28. Righini A, Zirpoli S, Parazzini C, Bianchini E, Scifo P, Sala $\mathrm{C}$, et al: Hippocampal infolding angle changes during brain development assessed by prenatal MR imaging. AJNR Am J Neuroradiol 27:2093-2097, 2006

29. Sato N, Hatakeyama S, Shimizu N, Hikima A, Aoki J, Endo $\mathrm{K}$ : MR evaluation of the hippocampus in patients with congenital malformations of the brain. AJNR Am J Neuroradiol 22:389-393, 2001

30. Seo JH, Holland K, Rose D, Rozhkov L, Fujiwara H, Byars A, et al: Multimodality imaging in the surgical treatment of children with nonlesional epilepsy. Neurology 76:41-48, 2011

31. Shinnar S, Bello JA, Chan S, Hesdorffer DC, Lewis DV, Macfall J, et al: MRI abnormalities following febrile status epilepticus in children: the FEBSTAT study. Neurology 79:871-877, 2012

32. Uematsu A, Matsui M, Tanaka C, Takahashi T, Noguchi K, Suzuki M, et al: Developmental trajectories of amygdala and hippocampus from infancy to early adulthood in healthy individuals. PLoS One 7:e46970, 2012

33. Utsunomiya H, Takano K, Okazaki M, Mitsudome A: Development of the temporal lobe in infants and children: analysis by MR-based volumetry. AJNR Am J Neuroradiol 20:717723,1999

34. Vannest J, Rajagopal A, Cicchino ND, Franks-Henry J, Simpson SM, Lee G, et al: Factors determining success of awake and asleep magnetic resonance imaging scans in nonsedated children. Neuropediatrics 45:370-377, 2014

35. Voets NL, Bernhardt BC, Kim H, Yoon U, Bernasconi N: Increased temporolimbic cortical folding complexity in temporal lobe epilepsy. Neurology 76:138-144, 2011

36. Wieser HG, Blume WT, Fish D, Goldensohn E, Hufnagel A, King D, et al: ILAE Commission Report. Proposal for a new classification of outcome with respect to epileptic seizures following epilepsy surgery. Epilepsia 42:282-286, 2001

\section{Disclosures}

The authors report no conflict of interest concerning the materials or methods used in this study or the findings specified in this paper.

\section{Author Contributions}

Conception and design: Leach, Greiner, Mangano. Acquisition of data: Leach, Awwad, Greiner, Mangano. Analysis and interpretation of data: Leach, Awwad, Greiner, Vannest, Miles. Drafting the article: Leach, Greiner, Mangano. Critically revising the article: all authors. Reviewed submitted version of manuscript: all authors. Approved the final version of the manuscript on behalf of all authors: Leach. Statistical analysis: Leach. Administrative/ technical/material support: Leach. Study supervision: Leach.

\section{Correspondence}

James L. Leach, Department of Radiology, Cincinnati Children's Hospital Medical Center, 3333 Burnet Ave., Cincinnati, OH 45226. email: james.leach@cchmc.org. 